\title{
MEASUREMENT OF MAGNETIC CORRELATION EFFECTS IN PARTICULATE RECORDING MEDIA
}

\author{
R. Rosman ${ }^{\dagger}$, M.D. Clarke ${ }^{\ddagger}$, M.Th. Rekveldt ${ }^{\dagger}$, P.R. Bissell ${ }^{\ddagger}$ and R.W. Chantrell ${ }^{\ddagger}$ \\ † Interfacultair Reactor Instituut, Technical University Delft, The Netherlands. \\ $\ddagger$ Magnetic Materials Research Group, Lancashire Polytechnic, Preston PR1 2TQ, U.K.
}

\begin{abstract}
Noise and neutron depolarisation (ND) measurements on a particulate recording tape, at various remanent states through the dc demagnetisation remanence curve, are presented. The microstructure of the tape is discussed in relation to both media noise and a correlation length $\xi$, derived from a ND experiment. The existence of density variations and superdomains elongated along the applied field direction (length direction of the tape) is deduced.
\end{abstract}

\section{INTRODUCTION}

More detailed knowledge concerning the physical and magnetic microstructures of particulate recording media is necessary to explain the discrepancy between the theoretically predicted and the experimentally determined noise characteristics of these media. In particular, magnetic correlations due to interaction effects and particle agglomerations within the medium are known to strongly affect these characteristics $[1,2,3]$. Therefore it is important to have access to techniques, other than noise measurements, which can study both the physical and magnetic microstructures of recording media. A technique which has the possibility to yield detailed information about the microstructures of a magnetic medium is the neutron depolarisation (ND) technique. Although this technique has been known for quite some time $[4,5]$, it has only recently been applied to the study of recording media [6].

The polarisation vector of a polarised neutron beam is analysed after transmission through a magnetic medium in the ND technique. During transmission the polarisation vector is affected by magnetic inhomogeneities in the medium: mean magnetic induction results in a net rotation of the polarisation vector while fluctuations in the magnetic induction results in an effective shortening of the polarisation vector (depolarisation). In general, a ND experiment yields a mean magnetic induction $\underline{B}$, the local magnetic texture and a mean correlation length along the neutron path. This paper will be confined to the interpretation of the latter, defined as [7]

$$
\xi=\frac{2}{\Delta V} \int_{\Delta V} d^{3} x \int_{z_{0}}^{z} d z^{\prime} \underline{B}^{f}(x, y, z) \cdot \underline{B}^{f}\left(x, y, z^{\prime}\right) /\left[\bar{\epsilon}\left(\mu_{0} M_{s}\right)^{2}\right]
$$

Here, $\underline{B}^{f}(\underline{r})=\underline{B}(\underline{r})-\underline{\bar{B}}, \underline{B}(\underline{r})$ is the local magnetic induction, $\Delta V$ the volume of a representative part of the medium with thickness $\Delta L, \vec{\epsilon}$ the average particle volume fraction and $M_{s}$ the spontaneous particle magnetisation. The propagation direction $\left(\underline{e}_{0}\right)$ of the neutron beam is along $z$. Note that $\xi$ is given as a volume reduced length over which fluctuations in magnetic induction from its mean are correlated.

The value of $\xi$ is affected by many aspects of both the physical and magnetic microstructures, the most important of which are the intrinsic particle properties (size, shape \& material), interparticle correlations and density variations [8]. In an ensemble of uncorrelated particles with $\bar{B}=0, \xi$ is given by

$$
\xi=\frac{V}{4 \pi^{2}} \int_{S} d^{2} \underline{s}<\left(\underline{n}^{*}(\underline{\tilde{s}})\right)^{2} F(\underline{s})^{2}>
$$

with $V$ the particle volume, $\underline{s}$ the reciprocal position vector, $F(\underline{s})$ the particle formfactor, $\underline{\tilde{s}}=\underline{s} /|\underline{s}|, \underline{n}^{*}(\underline{s})=\underline{\tilde{s}} \times(\underline{n} \times \underline{\tilde{s}}), \underline{n}$ the unit vector along the particle magnetisation, $\langle>$ an average over all particles and $S$ the $x y$-plane of $\underline{s}$. For uncorrelated particles, $\xi$ approximates to the average particle size along $e_{0}$. When $\bar{B} \neq 0$, an increase in $\bar{B}$ results in a decrease in $B^{f}(\underline{r})$ and therewith in a trivial decrease in $\xi$. This decrease is proportional to the square of the reduced remanent magnetisation $m$ and depends on $\epsilon$ among other things. Positive (negative) correlations between the magnetisation orientations of neighbouring particles along $\underline{e}_{0}$ result in an increase (decrease) of $\xi$. Density variations may increase $\xi$ in the case $\bar{B}=0$. Then, the part of the medium with a particle density equal to $\bar{\epsilon}+\Delta \epsilon$ acts as a large magnetic inhomogeneity with magnetic amplitude $\Delta \epsilon \mu_{0} m M_{s}$.

This paper deals with the results of noise and ND measurements on a particulate recording tape as it is taken through the dc demagnetisation remanence curve, i.e., the sample is initially saturated and then measured at various remanent states after applying progressively larger $\mathrm{dc}$ fields in the opposite sense. These results will also be discussed in relation to results taken after ac bulk erasure.

\section{EXPERIMENTAL}

The tape samples used in the ND and noise experiments were all taken from a commercial Co modified $\gamma-\mathrm{Fe}_{2} \mathrm{O}_{3}$ audio tape. The particles are elongated, with a diameter $(a)$ between 30 and 80 $\mathrm{nm}$ and a length $(b)$ between 0.2 and $0.5 \mu \mathrm{m}$ [9]. The tape had been initially characterised magnetically using a VSM. To establish saturation magnetisation and the dc demagnetised remanent states, the tapes were passed through a solenoid producing an in plane magnetisation along the length direction of the tape ( $y$ direction). A commercially available bulk eraser was used to produce the bulk erased state. A standard audio cassette transport system and head were used for the noise measurements, where the voltage induced at the head was measured on a noise meter. The ND experiments were performed on a set up described in [6].

\section{RESULTS \& DISCUSSION}

Figure 1 gives the correlation length perpendicular to the plane of the tape ( $z$-direction), $\xi_{p}$, versus the reduced magnetisation $m$ at various remanent states through the dc demagnetisation remanence curve. It also includes a measurement taken after ac bulk erasure. The value of $\xi_{p}$ in the dc demagnetised states can be characterised by a slight decrease with increasing magnetisation away from $m=0$.

If the particle orientation is assumed to be uniaxial around the $y$-axis, with the angle between the particle longitudinal axis 
FIGURE 1. Correlation length perpendicular to the tape length versus reduced magnetisation.

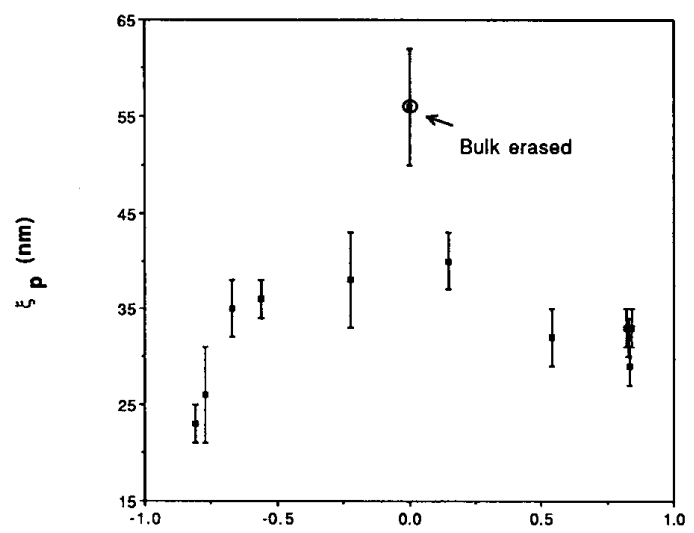

Reduced Magnetisation

FIGURE 2. Angular dependence of correlation length.

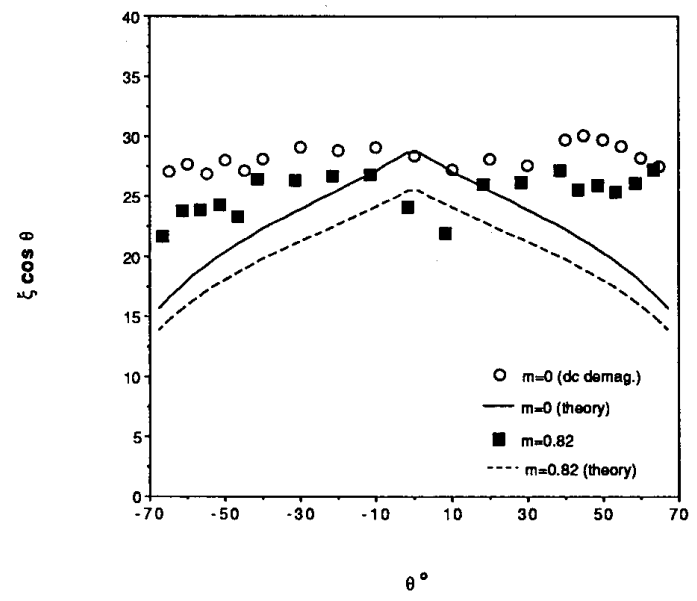

and the $y$-axis following a Gaussian distribution with standard deviation $\sigma$, then the maximum value of $m=0.84$ corresponds to $\sigma=24^{\circ}$. From this orientational texture function and a given value of the particle diameter, it is possible to calculate the correlation length using Equation 2. The value of diameter giving rise to the measured value of $\xi_{p}$ in zero field is $a=45 \mathrm{~nm}$. This value is in the order of the particle diameter. Consequently, it is reasonable to suppose that the correlations in the $z$-direction are at the most weak.

The interpretation of $\xi_{p}$ in the bulk erased tape sample is more complex and is the subject of continued investigations.

To study non-perpendicular effects, the propagation direction of the beam was varied in the $y z$-plane with $\theta$ being the angle between $\underline{e}_{0}$ and the $z$-axis. Figure 2 gives these experimental results (symbols) and also uncorrelated theory values (lines) for $\xi \cos \theta$ versus $\theta$ at the $m=0$ (dc demagnetised) and at the $m=0.82$ states. The uncorrelated theory values are numerically calculated from Equation $2\left(\sigma=24^{\circ}, a=38 \mathrm{~nm}, b / a=10\right)$, decreasing with increasing $\theta$. The actual lines are rather insensitive for the exact value of $b / a$ as long as $b / a>5$. For $b / a<5$ the decrease
FIGURE 3. Media noise as a function of remanent magnetisation.

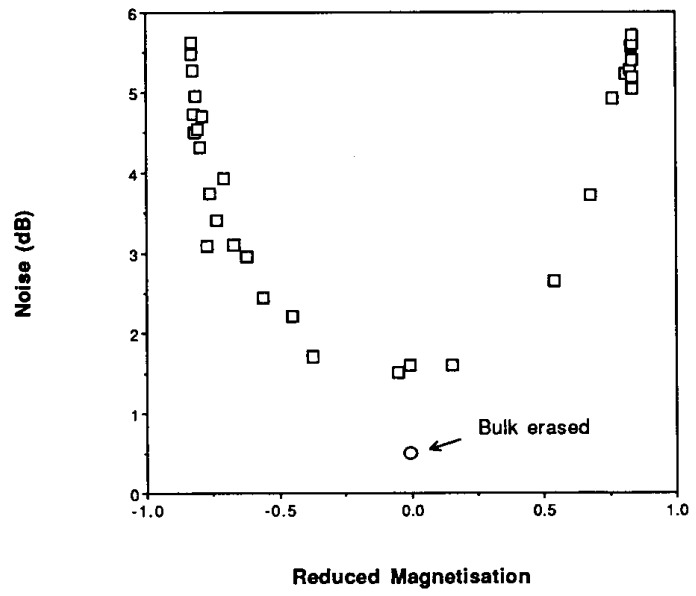

of $\xi \cos (\theta)$ with increasing $\theta$ is even stronger. The determined $\xi$ clearly does not correspond to uncorrelated particles. At large $\theta$ an additional correlation length is seen, up to a value of $17 \mathrm{~nm}$.

Elongated superdomains, oriented closely around the $y$ - direction, is a possible reason for this additional correlation length. A superdomain should be considered as a subvolume of the tape, the mean magnetisation of which may differ from the mean magnetisation of the tape. Such superdomains yield a $\xi$ proportional to $1 / \cos (\theta)$. This can either be calculated from Equation 2 or follows from the consideration that the size of these superdomains along $\underline{e}_{0}$ is inversely proportional to $\cos (\theta)$. The $y$-components of the particles in the $m=0.82$ tape have nearly all the same sign ( $m$ almost has its maximum value). Then the superdomains correspond to subvolumes in which the mean particle orientation differs from the mean particle orientation within the tape or in which the density strongly differs from its mean value [8]. For the $m=0$ state, the superdomains should be interpreted as switching volumes elongated along the field direction. Such switching volumes are predicted theoretically [10] and found experimentally in powders [11].

Little difference is seen between the $\theta$-dependence of $\xi$ of the $m=0$ and the $m=0.82$ tape. This does not indicate that the size of the superdomains in the tapes are equal. Following from the value of $\xi_{p}$ in both tapes the superdomains should contain approximately only 1 particle along $z$. However, the size along $y$ may change with $m$. The measurements presented in Figure 2 are very insensitive for the exact length of the superdomains along $y$ if this length exceeds $15 a$. In order to deduce the exact value of this length, $\xi$ should be measured at $\theta=90^{\circ}$, which is not possible.

Figure 3 shows the total media noise power (background noise having been subtracted) versus reduced magnetisation at various remanent states through the dc demagnetisation curve. The graph is characterised by a minimum in the dc erased state, with maximum noise at the saturated states. The way in which the noise increases with increasing $m$ will be related to density variations and the forming of superdomains [12]. With increasing $m$, the contribution of a single density variation increases and a larger part of the coating contributes to the noise. The contribution of superdomains to the noise increases with increasing superdomain volume. It is very difficult to separate the contributions of density variations and superdomains. 
At $m=0$ density variations do not contribute to the noise. The fact that the noise in the bulk erased state is much lower than the noise in the dc demagnetised state may indicate that the volume of possible superdomains in the former state are smaller.

The ND experiments yields a $\xi_{p}$ which has at most a weak dependence on $m$ during $\mathrm{dc}$ demagnetisation process. On the other hand, the noise power appears to be highly dependent on $m$. This difference may be due partly to the fact that the noise technique is much more sensitive to small density variations than the ND technique. It can also be the result of a superdomain size along $y$ which increases with increasing $m$. The quantity $\xi_{p}$ is sensitive to the size of the superdomains along $\underline{e}_{0}$ only, while the noise power is sensitive to the superdomain volume.

\section{CONCLUSIONS}

The ND correlation length perpendicular to the plane of the tape is of the order of the particle size at all values $m$ of the remanent magnetisation after dc demagnetising along the length direction of the tape, although a weak variation in $\xi$ with $m$ is also observed. From the ND experiments, superdomains elongated along the direction of the applied field have been deduced. An increase of the noise power with increasing $m$ has been found. This increase may be due to small density variations within the coating of the tape or to an increase in the size of the superdomains along the length direction of the tape with increasing $m$. The existence of superdomains of particles correlated along the field direction is revealed by the departure of $\xi$ with angle from uncorrelated theory. The magnetic microstructure after bulk erasing clearly differs from the magnetic microstructure after dc demagnetising. Further work is in progress to investigate this effect.

\section{ACKNOWLEDGEMENTS}

This work was supported by the CAMST (Community Action on Magnetic Storage Technology) project of the EC science programme.

\section{REFERENCES}

[1] L. F. G. Thurlings IEEE Trans. Magn., vol. MAG-19, pp. 84-89, 1983.

[2] R. A. Arratia and H. N. Bertram, IEEE Trans. Magn. vol. MAG-20, pp. 412-420, 1984.

[3] M. Fearon, R. W. Chantrell, A. Lyberatos, and E. P. Wohlfarth, IEEE Trans. Magn. vol. MAG-23, pp. 174-176, 1987.

[4] G. M. Drabkin, E. I. Zabidarov, Y. A. Kasman, and A. I. Okorokov JETP, 29, (2), p. 261, 1969.

[5] M. Th. Rekveldt Z.Physik, vol. 259, pp. 391-410, 1973.

[6] R. Rosman, M. Th. Rekveldt, and H. A. J. Cramer J. de Physique, vol. C8, no. 12, 1988.

[7] R. Rosman and M. Th. Rekveldt Z. Phys. B, Jan. 1990.

[8] R. Rosman and M. Th. Rekveldt, to be presented at INTERMAG 90, cr-19.

[9] F. Jorgensen, The Complete Handbook of Magnetic Recording. Tab Books, 3rd ed., p. 264, 1988.

[10] M. Fearon, R. W. Chantrell, and E. P. Wohlfarth, J. Magn. Magn. Mater. 1990. (In press).

[11] R. Rosman and M. Th. Rekveldt, to be published.

[12] M. D. Clarke, P. R. Bissell and R. W. Chantrell, to be published. 